\title{
Copper Cu 64-DOTA-ECL1i
}

National Cancer Institute

\section{Source}

National Cancer Institute. Copper Cu 64-DOTA-ECL11. NCI Thesaurus. Code C158983.

A radiotracer composed of ECL1i (extracellular loop 1 inverso; $\mathrm{d}($ LGT FLKC)), an allosteric peptidic modulator of CC chemokine receptor type 2 (CCR2), conjug ated with 1,4,7,10tetra-azacyclododecane-1,4,7,10-tetra-acetic acid (DOTA) and labeled with the radionuclide copper $\mathrm{Cu} 64$, with potential imaging activity upon positron emission tomography (PET). Upon administration, the ECL1i moiety targets and allosterically binds to CCR2 expressing tumor cells. Upon PET imaging, the copper Cu 64 moiety can be visualized, thereby allowing the quantification of CCR2-expressing cells. CCR2, a G-protein coupled receptor expressed on the surface of monocytes and macrophages, stimulates the migration and infiltration of these cell types, and plays a significant role in angiogenesis, inflammation, tumor cell migration, and proliferation. Quantification of CCR2 may help predict chemotherapy resistance and identify early metastatic disease in certain cancers. 